\title{
Measurement of Drug-taking Compliance Based on RFID Technology
}

\author{
Zhao Xu Ren \\ Department of Science and Technology, Chengdu Technological University, Chengdu 611730, \\ China \\ wiston_ren@sina.cn
}

Keywords: Radio Frequency Identificaion (RFID); Drug-taking Compliance; Intel Wireless Sensor Platform (WISP)

\begin{abstract}
A high level of non-compliance relating to taking of medication can lead to compromised health benefits and wasted money. Improving patient compliance has the potential for improving issues related to Cardiovascular Disorder (CVD) and many other diseases. This paper developed a prototype system for monitoring medication dosages in a home health care environment. The combination of an RFID technology - the Intel Wireless Sensor Platform(WISPs) and the construction of a specific pill dispensing container was used in this prototype demonstrated that it is possible to use RFID technology to effectively and ubiquitously monitor and track drug-taking compliance. With further refinements on the dispensing unit and optimisations in the software, this system could help increase compliance and reduce health related issues.
\end{abstract}

\section{Introduction}

A high level of non-compliance (or non-adherance) in regards to taking prescription medicine is a cause of compromised health benefits and wasted money. A large review in 2001 [1] indicated that $30 \%-50 \%$ of patients fail to comply completely with treatment. Compliance issues have become a major focus for research and many interventions have taken place to help reduce the impact of non-compliance. Improving patient compliance has a great potential for improving issues related to Cardiovascular Disorder (CVD) and many other important chronic diseases. There are a number of ways that people can fail to comply with prescribed treatment, from whether they actually fill the prescription to whether they take the medication according to the prescribed regimen. This study is concerned with "secondary non-compliance", that is when patients have physical possession of the medication but do not follow the instructions exactly. Studying secondary non-compliance is difficult as this generally occurs within the patient's own home and it is not reflected in statistics that are routinely collected e.g. in terms of prescriptions filled.

There have been many developments in information technology intended to allow ubiquitous monitoring of a patient's health in their own homes, however demonstration of benefit is still elusive [2]. These approaches may be particularly beneficial for patients who have to live on their own and/or patients who can't make regular trips to the hospital $[3,4]$.

\section{Proposed prototype system}

RFID Technology. Radio Frequency Identification (RFID) has become an integral part of our lives, in many application areas [5]. RFID is a short-range radio technology that is best suited for communicating digital information about specific objects. In a typical scenario there are multiple RFID Tags/Transponders which are small and inexpensive and only one RFID Reader/Interrogator. Information can be sent from the tags by generating, modulating and transmitting a radio signal the reader can detect. A number of modulation techniques can be used to enable read-only or read-write communication.

Wireless Identification and Sensing Platform (WISP). WISP [6] is a new RFID tag technology that introduces a power model that is different to standard passive or active tags. WISPs are similar to passive tags in the sense that they only use power harvested from the reader's RF signals, and like 
active tags they can continue collecting data away from readers. This ability is achieved by storing the harvested energy in a capacitor for later use. This is particularly useful for applications that have limited contact with readers. WISPs have been designed to run on the standardised EPC Gen 2 infrastructure. (www.epcglobalinc.org). This allows the use of commercially available readers that are compatible with EPC Gen 2 tags to collect tag data from the WISP (Figure 1).

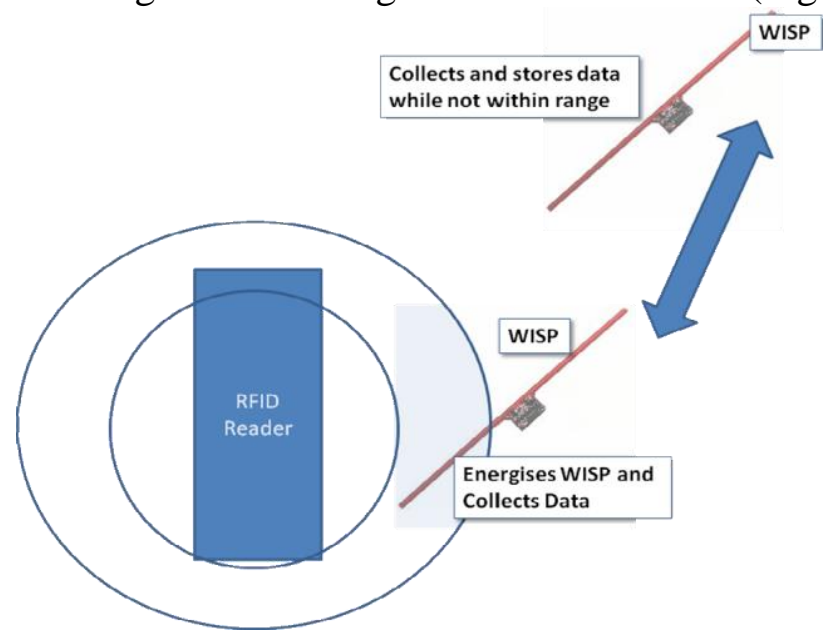

Fig. 1 WISP mode of action

Prototype development. The prototype was designed to allow reliable recording of the number of pills dispensed from a container. The prototype pill counter was built based on the following requirements:

Each pill being dispensed would be detected, and movement of the container that did not result in a pill being dispensed would not result in a false count

Because of limitations in the development environment for the WISP on-board computer, all data collection will occurr within range of the RFID reader, with minimal on-board processing.

Pill dispensing should be based on a simple operation or set of operations.

The development of a physical container is required to demonstrate the ability to dispense pills in such a way that it can be monitored and tracked using the WISP technology. The 3D accelerometer sensor was used to detect movement in the container whether it be a specific pattern or a difference in values from two or more WISPs. There was also the possibility of using a combination of the sensors such as using the temperature sensor to detect a hand and the 3D accelerometer to detect movement. However, another approach based on a lolly dispenser that had a more complex mechanism yet was still low cost seemed promising. We used the PivoTell Advance Dispenser as seen in Figure 2.

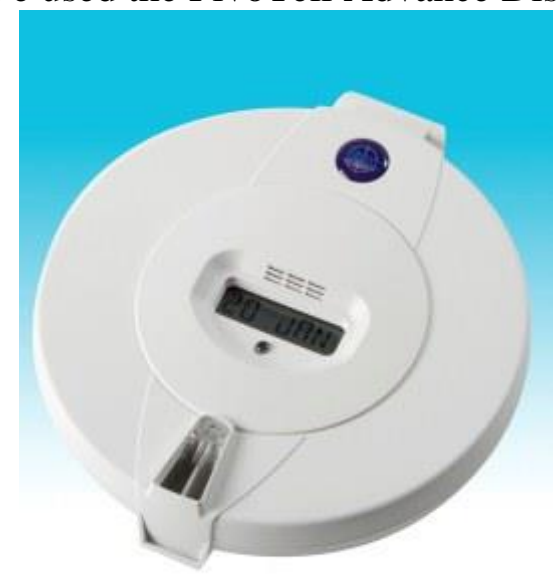

Figure. 2 PivoTell Advance Dispenser.

This design could not utilise a single WISP for movement. The incorporation of two WISP tags allows the application to detect dif- ferences between their values, so when the button is pressed one 
of the WISPs rotates with respect to the other. The dif- ference in values is detected by the system and if the difference is great enough it can be inferred that a pill was dis- pensed. The angle "noise" of the accelerometer is +/- 0.6 of a degree when stationary so the amount of rotation has to be greater than 2 degrees to reliably determine that the button was pushed. Any gross movement of the container will be picked up by both WISPS and hence will be cancelled out in the processing phase.

To achieve the ability to rotate the rotating WISP we built an internal mechanism that forces the WISP to rotate around the point specified in Figure 3. The spring ensures the WISP locks back into place when the button is released; the bar is used for holding one side of the WISP up while the other drops. A second WISP tag is placed anywhere on the con- tainer and calibrated to return the same values during gross movement of the whole devices as the rotating tag. This design can handle being dropped and shaken around as the detection is based on differences rather than individual movements.
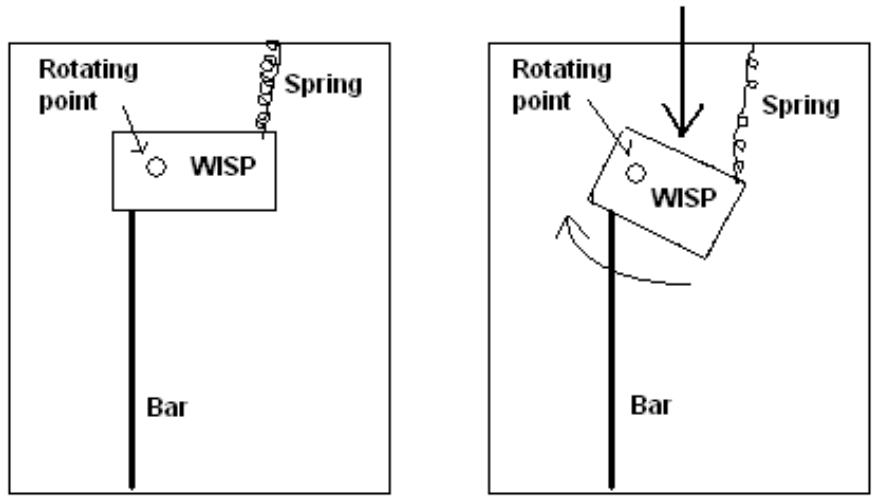

Figure. 3 How the internal tilting mechanism works. (only one WISP shown for clarity)

Some considerations taken into account were isolating the sensors from elements that might affect reliability of results and distinguishing between actual dispensing of pills and general handling of the pill container.

The software development was fairly simple involving modules to calibrate the two WISPs, a module to read the unique tag IDs and record the angle as derived from the onboard sensors, and a calculation module to calculate the difference between the two angles and count pills dispensed. The "pills dispensed" algorithm is based on an angle difference of more than 6 degrees occurring, and then returning to less than this figure.

\section{Results}

For testing the system as a whole (including container, static and dynamic tags, software and users) a number of tasks were used to ensure the system provided reliable results in all situations. The data collected from each test included a test number, software detected result (binary), actual result (binary), tilt value of static tag, tilt value of dynamic tag and the tilt difference. When evaluating the results the software detected result and the actual result pair were studied to see how closely the samples agree.

The initial test was completed with a known number of pills using very deliberate movements when pushing the button. This was designed to test the functionality of the container and software when being used in ideal conditions. Each test was performed separately with values being recorded after each press. Results are shown in Table 1 for:

Detected Result: Whether or not the software detected that a pill had been dispensed.

Actual Result: If a pill was dispensed from the device.

Tilt Static Chip: The tilt value returned from the WISP for the tag that does not move.

Tilt Dynamic Chip: The tilt value returned from the WISP for the tag that rotates when the button is pressed.

Difference Tilt: The difference between the tilt values. Used for determining if the button has been pushed. 
The second stage of testing the application and pill dispensing container was to get three people to use the device without giving them direct instructions on how it works. They were instructed to dispense five pills (lollies) at a random interval. The attempts from each user can be seen in Table 2 .

Table 1 Initial calibration

\begin{tabular}{|c|c|c|c|c|c|}
\hline Test & $\begin{array}{l}\text { Detected } \\
\text { Result }\end{array}$ & $\begin{array}{l}\text { Actual } \\
\text { Result }\end{array}$ & $\begin{array}{l}\text { Tilt Static } \\
\text { Chip }\end{array}$ & $\begin{array}{l}\text { Tilt Dynamic } \\
\text { Chip }\end{array}$ & $\begin{array}{c}\text { Difference } \\
\text { Tilt }\end{array}$ \\
\hline 1 & 1 & 1 & 421 & 383 & 38 \\
\hline 2 & 1 & 1 & 430 & 383 & 47 \\
\hline 3 & 1 & 1 & 415 & 379 & 36 \\
\hline 4 & 1 & 1 & 426 & 385 & 41 \\
\hline 5 & 1 & 1 & 431 & 394 & 37 \\
\hline 6 & 1 & 1 & 422 & 390 & 32 \\
\hline 7 & 1 & 1 & 426 & 382 & 44 \\
\hline 8 & 1 & 1 & 429 & 385 & 44 \\
\hline 9 & 1 & 1 & 435 & 399 & 36 \\
\hline 10 & 1 & 1 & 431 & 387 & 44 \\
\hline \multicolumn{6}{|c|}{ Table 2 Test user results } \\
\hline Test & $\begin{array}{l}\text { Detected } \\
\text { Result }\end{array}$ & $\begin{array}{l}\text { Actual } \\
\text { Result }\end{array}$ & $\begin{array}{l}\text { Tilt Static } \\
\quad \text { Chip }\end{array}$ & $\begin{array}{l}\text { Tilt Dynamic } \\
\text { Chip }\end{array}$ & $\begin{array}{c}\text { Difference } \\
\text { Tilt }\end{array}$ \\
\hline 11.1 & 1 & 1 & 424 & 386 & 38 \\
\hline 11.2 & 1 & 1 & 425 & 392 & 33 \\
\hline 11.3 & 1 & 1 & 424 & 378 & 46 \\
\hline 11.4 & 1 & 1 & 423 & 393 & 30 \\
\hline 11.5 & 1 & 1 & 427 & 387 & 40 \\
\hline 12.1 & 1 & 1 & 421 & 387 & 34 \\
\hline 12.2 & 1 & 1 & 418 & 387 & 31 \\
\hline 12.3 & 1 & 1 & 422 & 380 & 42 \\
\hline 12.4 & 1 & 1 & 419 & 381 & 38 \\
\hline 12.5 & 1 & 1 & 421 & 383 & 38 \\
\hline 13.1 & 1 & 1 & 381 & 346 & 35 \\
\hline 13.2 & 1 & 1 & 382 & 350 & 32 \\
\hline 13.3 & 1 & 1 & 381 & 349 & 32 \\
\hline 13.4 & 1 & 1 & 379 & 344 & 35 \\
\hline 13.5 & 1 & 1 & 385 & 342 & 43 \\
\hline
\end{tabular}

\section{Conclusions}

The results from the tests show a completely reliable software detection result. This result was obtained from a series of four users including one of the authors. After plotting and analysing the data it was clear to see that all the results form a linear pattern that is determined by the degree of tilt of the device. The correlation result of 0.97 showed a very strong positive relationship between the static and dynamic tilt variables, during dispensing.

The combination of RFID technology (WISPs) and the construction of a specific pill dispensing container in this prototype has proved to demonstrate that it is possible to use RFID technology to effectively and ubiquitously monitor and track drug taking compliance. With further refinements on 
the dispensing unit and optimisations in the software this product could be manufactured and released to home care patients to help increase compliance and reduce health related issues.

\section{References}

[1] J. van der Geer, J.A.J. Hanraads, R.A. Lupton, The art of writing a scientific article, J. Sci. Commun. 163 (2000) 51-59.

[2] Vermeire E., Patient adherence to treatment: three decades of research. A comprehensive review. J Clin Pharm Ther. 26 (2010) 331 - 342.

[3] Martin S., Smart home technologies for health and social care support. Cochrane Database of Systematic Reviews, 2008.

[4] Choi JM, et al. A System for Ubiquitous Health Monitoring in the Bedroom via a Bluetooth Network and Wireless LAN. in Engineering in Medicine and Biology Society, 2004. IEMBS '04. 26th Annual International Conference of the IEEE. 2004.

[5] Otto C, et al., System architecture of a wireless body area sensor network for ubiquitous health monitoring. Journal of Mobile Multimedia. 1 (2009) 307-326.

[6] Landt J, The history of RFID Potentials, IEEE, 2005.

[7] Sample AP, et al., Design of an RFID-Based Battery-Free Programmable Sensing Platform. Instrumentation and Measurement. IEEE Transactions on Signals and Systems, 57 (2008) 2608-2615.

[8] Houliston B, Merry A, and Parry DT, Sensors and Insensibility: Monitoring Anaesthetic Activity with RFID, in Health Informatics New Zealand Forum. 2013, HINZ: Rotorua.

[9] Gentry T, Smart homes for people with neurological disability: State of the art. NeuroRehabilitation. 25 (2012) 209-217. 